\title{
Recent Advances in Social Network Analysis, Management and Security
}

\section{J.UCS Special Issue}

\author{
Yaser Jararweh \\ (Jordan University of Science and Technology, Irbid, Jordan \\ yijararweh@just.edu.jo) \\ Mohammad Al-Smadi \\ (Jordan University of Science and Technology, Irbid, Jordan \\ masmadi@just.edu.jo) \\ Elhadj Benkhelifa \\ (Staffordshire University, Stafford, United Kingdom \\ e.benkhelifa@staffs.ac.uk)
}

\begin{abstract}
Social network analysis is concerned with the study of relationships between social entities. The recent advances in internet technologies and social media sites, such as Facebook, Twitter and LinkedIn, have created outstanding opportunities for individuals to connect, communicate or comment on issues or events of their interests. Social networks are dynamic and evolving in nature; they also involve a huge number of users. Frequently, the information related to a certain concept is distributed among several servers. This brings numerous challenges to researchers, particularity in the data mining and machine learning fields. Privacy and security in Social networks are critical concerns especially with the case of cross-platform security. Despite the tremendous research conducted around social networks, literature shows that challenges in the application of social networks analysis in different fields and security of social networks are still need to be addressed.

The purpose of this special issue was to provide a forum for researchers to present and discuss their work which is related to social network analysis, management and security. It aims to investigate the opportunities in all aspects of Social Networks Analysis, Management and Security. In addition, it seeks for novel contributions that help mitigating possible challenges. The special issue includes, among other submissions from the open call for papers, extended versions of accepted papers from the Fifth International Conference on Social Networks Analysis, Management and Security(SNAMS-2018) that took place in Valencia, Spain. during October 15-18, 2018. In response to this special issue, we received a total of 15 submissions, these submissions were reviewed by domain experts who recommended 8 papers to be accepted and to be included in this special issue. The 8 accepted articles are summarized as follows.
\end{abstract}


The first article, authored by Henrique Damasceno Vianna and Jorge Luis Victória Barbosa, entitled as "Pompilos, a Model for Augmenting Health Assistant Applications with Social Media Content". The authors performed a random experiment during one month and a half on two groups to assess the influence of messages related to the prevention of chronic diseases. Those messages presented information on a healthier diet, the practice of physical activities, and ways to lose weight, from monitored Twitter profiles on the habits of health assistant web application's users.

In the second article: "An Intelligent Recommender System Based on Association Rule Analysis for Requirement Engineering", the authors Mohammad Muhairat, Shadi ALZu'bi, Bilal Hawashin, Mohammad Elbes and Mahmoud Al-Ayyoub, proposed an intelligent recommender system for requirement engineering based on association rule analysis, which is a main category in Data Mining. Such recommender would contribute in enhancing the accuracy of the gathered requirements and provide more comprehensive results. Conducted experiments in this work prove that Frequent Pattern Growth outperformed Apriori in terms of execution and space consumption, while both methods were efficient in term of accuracy.

In the third article "Scalable Distributed Metadata Server Based on Nonblocking Transactions", co-authored by Qanita Bani Baker, Farah Shatnawi, Saif Rawashdeh, Mohammad Al-Smadi and Yaser Jararweh, in their paper entitled "Detecting Epidemic Diseases Using Sentiment Analysis of Arabic Tweets" proposed a new approach in order to detect Influenza using machine learning techniques from Arabic tweets in Arab countries. This paper is the first study of epidemic diseases based on Arabic language tweets. The authors have collected, labeled, filtered and analyzed the influenza-related tweets written in the Arabic language. Several classifiers were used to measure the quality and the performance of the approach, which are: Naive Bayes, Support Vector Machines, Decision Trees, and K-Nearest Neighbor.

The fourth article: "Label Clustering for A Novel Problem Transformation in Multi-label Classification", co-authored by Smail Sellah and Vincent Hilaire, focused on the multi-label classification, more precisely those methods that transforms a multilabel classification into a single label classification. The authors proposed a novel problem transformation that leverage label dependency. They used Reuters-21578 corpus that is among the most used for text categorization and classification research.

In the fifth article, the authors, Kohei Hiraga, Osamu Tatebe and Hideyuki Kawashima, proposed a design of a scalable distributed metadata server, PPMDS, for parallel file systems using multiple key-value servers. In PPMDS, hierarchical namespace of a file system is efficiently managed by multiple servers. Multiple entries can be atomically updated using a nonblocking distributed transaction based on an algorithm of dynamic software transactional memory. Performance evaluation shows the scalable performance up to 3 servers, and achieves 62,000 operations per second, which is $2.58 \mathrm{x}$ performance improvement compared to a single metadata performance.

In the sixth article: "User-Oriented Approach to Data Quality Evaluation", the authors, Anastasija Nikiforova, Janis Bicevskis, Zane Bicevska and Ivo Oditis, proposed a new data object-driven approach to data quality evaluation. It consists of three main components: (1) a data object, (2) data quality requirements, and (3) data quality evaluation process. All of the three components of the presented data quality model are described using graphical Domain Specific Languages (DSLs). In accordance with Model-Driven Architecture (MDA), the data quality model is built in 
two steps: (1) creating a platform-independent model (PIM), and (2) converting the created PIM into a platform-specific model (PSM).

In the seventh article: "A Framework for Online Social Network Volatile Data Analysis: A Case for the Fast Fashion Industry", co-authored by Anoud Bani Hani, Feras Al-Obeidat, Elhadj Benkhelifa, and Oluwasegun Adedugbe, investigated the effectiveness of current data mining techniques when used to identify consumer satisfaction towards fast fashion products. This is carried out by designing, implementing and testing a software artefact that utilises data mining techniques to obtain, validate and analyse fast fashion social data, sourced from Twitter, to identify consumer satisfaction towards specific product types. In addition, further analysis is carried out using a sentiment scaling method adapted to the characteristics of fast fashion.

The eighth article is authored by Patrick Juola and entitled as "Authorship Studies and the Dark Side of Social Media". Juola discusses the technology, focusing on its application to social media in a variety of disciplines. It includes a brief survey of the history as well as three tutorial case studies, and discusses several significant applications and societal benefits that authorship analysis has brought about. It further argues, though, that while the benefits of this technology have been great, it has created serious risks to society that have not been sufficiently considered, addressed, or mitigated.

In summary, we would like to sincerely thank all the authors and the reviewers for their contributions and efforts invested to prepare these publications. We hope that all eight articles will be found interesting and valuable for the Social Networks Analysis researchers and all other interested readers. 